\title{
Elaborated plan for incoming surgical emergency management in Sub-Saharan Africa
}

\section{Olivier Kubwimana}

College of Medicine and Health Sciences, University of Rwanda, Kigali, Rwanda
Correspondence: Oliver Kubwimana Orthopedic Surgery, University of Rwanda, Kigali, Rwanda

Tel +250788 89643 I

Email oliviee3@gmail.com
This article was published in the following Dove Press journal: Open Access Emergency Medicine

Background: Globally, there has been an increase in injuries as a major cause of death. This burden is mainly due to an increase in road traffic injuries, and it poses an enormous burden in low- and middle-income countries. Musculoskeletal and head injuries are the most prevalent ones, which has led to an overcrowding of traumatic surgical emergencies.

Methodology: An unrestricted search was done in different research databases for articles published in English between January 2005 and November 2017 focusing on traumatic surgical emergencies.

Results: The past several decades on the global health landscape are notable for increases in traumatic surgical emergencies. Although this burden of surgical emergencies is universal, high-income countries have implemented measures including mass casualty incident management, which continuously reduces the mortality and morbidity of trauma-related injuries. Nonindustrialized countries are facing almost the same burden, but there is still a lack of enough sustainable measures for combating this burden. Nevertheless, the Rwandan pre-hospital emergency care service (SAMU) which integrate pre-hospital services, has contributed a lot in the management of emergencies in Rwanda. Limb and head injuries are increasing, and there is a trend that this would continue.

Conclusion: A global upward shift in traumatic surgical emergencies was noted for the past several decades. This trend will continue, and it poses a significant burden in Sub-Saharan Africa, along with worldwide. The Sub-Saharan African mortality and morbidity rates are increasing in regard to traumatic surgical emergencies. Task shifting, initiation of similar systems such as SAMU, increase in workforce, conducting enough research, mass casualty incident protocols implementation, and zero tolerance in regard to alcohol or psychoactive substances by road users are recommendations from this review.

Keywords: traumatic surgical emergencies, road traffic injuries, musculoskeletal and head injuries

\section{Background}

Globally, there has been an exponential shift in the major cause of death from infectious diseases to injury. According to the World Health Organization (WHO), injury constitutes $16 \%$ of disease global burden, whereby injury causes 5.8 million deaths globally and 78 million to remain morbid. ${ }^{1}$ Only $10 \%$ of the world population live in settings in line with laws against potential risk factors of road traffic injuries (RTIs). ${ }^{2}$ The WHO report on road traffic safety identifies RTI to be number one killer worldwide in the population between 15 and 29 years of age. ${ }^{3}$ RTI constitutes a global 
burden, which is more significant in low- and middle-income countries. Mortality rate due to RTI is higher in Africa at 24.1/100,000, whereas in Europe and America, it is, respectively, 10.3/100,000 and 16.1/100,000 per year. There is a tremendous increase in RTI in Sub-Saharan region; in Kenya, there was a fourfold increase in 30 years. A study done in Tanzania estimated a 44\% increase of RTI in 10 years. However, several studies conducted in this region revealed musculoskeletal injuries to be more prevalent in RTI patients. ${ }^{4}$ Afuwape et al, ${ }^{5}$ through a retrospective study, reported traumatic emergencies to be the second leading cause of death accounting for $31 \%$, with head injury and limb trauma being the prevalent form of injury. ${ }^{5}$ Low-income countries face an economic burden caused by RTI amounting to $1 \%$ of gross national product, as this amount goes to management of those injuries. ${ }^{6}$ Although Haiti is geographically part of the western world, the fact that it is a nonindustrialized country made the findings of Aluisio et $\mathrm{al}^{7}$ resemble the pattern of surgical emergencies seen in Sub-Saharan Africa. This crosssectional study reported extremity and head and neck injuries with road traffic accidents (RTAs) as the most prevalent form of injury, leading to trauma cases in public hospitals of Northeast region of Haiti. ${ }^{7}$ The aim of conducting this study was to reveal the commonly increasing traumatic surgical emergencies by reviewing the current literature in order to come up with some recommendations.

\section{Methodology of review Research question}

- What are the increasing traumatic surgical emergencies in Sub-Saharan Africa?

- How prepared is Sub-Saharan Africa in managing those cases?

\section{Research objectives}

Main objectives

- Assess the commonly increasing traumatic surgical emergencies.

- Evaluate the level of preparedness in regard to traumatic surgical emergencies in Sub-Saharan Africa.

\section{Specific objectives}

- Report the common traumatic surgical emergencies for the last decade.

- Document measures elaborated by emergency departments.

- Report public health measures tackling decreasing traumatic surgical cases.

\section{Scope of review}

"Search for commonly increasing traumatic emergency in SubSaharan Africa" in order to come up with recommendations.

\section{Search methodology}

An unrestricted search was conducted in several research databases; such as PubMed, Hinari, Embase, GoogleScholar, and Webmed. Articles published between January 2005 and February 2018 in English and focusing on pattern of traumatic surgical emergencies were selected for full text review and included in the study. In total, 21 articles that met the inclusion criteria were selected. Articles focusing on traumatic surgical emergencies in Sub-Saharan Africa were retained for the study; articles reporting specifically on surgical emergencies in high-income countries were also included for comparison with Sub-Saharan Africa. Moreover, public health-related articles focusing on measures reducing RTIs were also included.

\section{Results}

\section{Pattern of surgical emergencies during the last several decades}

High-income countries

In higher income countries, there have been dramatic increases in emergency cases during the last 10 years, although this is not specific to surgery. The USA has seen an annual increase of 5\%-11\%, while Germany has seen an increase of $16 \%$ from 2005 up to $2008 .{ }^{8}$ In the USA, based on a report by Emejulu et al, ${ }^{9}$ the RTAs as a cause of head injury accounted for $28 \%$ of cases. They concluded that despite advanced emergency management in high-income countries, head injury is a public health issue. ${ }^{9}$ DiMaggio et al, ${ }^{10}$ with a retrospective analysis, showed the trend of increased traumatic injuries in an elderly population from 2000 to 2011, which was economically significant as the hospital-related cost was more than double that for USA for 10 years. In Norway, a study done by Bogstrand et $\mathrm{al}^{11}$ reported that the presence of alcohol alone or in combination with other psychoactive substances in blood of patients admitted to the emergency ward for RTIs. Thus, implementation of road traffic-related measures should focus on reducing the use of alcohol and psychomotor substances among road users. ${ }^{11}$

\section{Sub-Saharan Africa}

In Rwanda, a study done by Aluisio et al ${ }^{12}$ demonstrated that limb trauma followed by head injury were the prevalent pediatric injuries received. Moreover, this study recommended establishment of effective measures for management 
of these injuries. ${ }^{12}$ This was also emphasized by Stephens et al ${ }^{13}$ through a study done in Uganda that limited resources settings significantly affect management of lower limb injury specifically femur and tibia fractures, as the study resulted in showing that volumes of cases outweighed available resources. ${ }^{13}$ Although a little is known about the pattern of injury in our region, there was a report in Rwanda where $7 \%$ of surgical admissions were due to injury; moreover, Yamuragiye et al, ${ }^{1}$ through a retrospective descriptive study, reported RTIs to be the main cause of injury and also reported that young males were the most vulnerable group. In Ethiopia, like in other low-income countries, the number of traumatic surgical cases is dramatically increasing, although most of the time there are no clear measures to combat this major issue. This study recommended establishing measures of RTA reduction in constrained settings. ${ }^{14}$ Chekijian et al,${ }^{15}$ though a review with high-level evidence, reported that RTIs occupied the 9th position on the list of global leading causes of death. Hence, they predict an ascending shift and state that RTIs would rank 5th in 2030.

\section{Measures that contributed to significant reduction of surgical emergencies in industrialized countries}

For the last 67 years in the USA, there has been a significant reduction in death post-RTA; to be more specific, from 2000 up to 2009 , a $10 \%$ decrease in fatality rate was noted. This resulted from initiation of strong prehospital services and the beginning of emergency medicine as a department, in addition to implementation of road traffic regulations and emergency medical services. Moreover, there was the establishment of an enormously strong institution in the last decade, the Centers for Disease Control and Prevention. ${ }^{15}$ Mass casualty incident is a challenging situation globally, and its effective management is based on preparedness of the team, hospital settings in terms of protocol and equipments, and good prehospital interventions. ${ }^{16}$

\section{Elaborate plan for incoming surgical emergency management in Sub-Saharan Africa}

The world report on RTI prevention was established in 2004, as a joint effort between WHO and World Bank, and it specifically targeted the global burden caused by RTIs. ${ }^{15,17}$ Moreover, there is an established joint effort by high-income countries of strengthening surgical practice and research in the developing world. ${ }^{18}$ Scott et a ${ }^{19}$ through a study done in Rwanda proved that the Rwandan prehospital emergency care service (SAMU) which integrate pre-hospital services, was extremely effective in management of traumatic surgical emergencies, specifically in terms of providing paramount prehospital care by giving the necessary initial management during the transport from the site of injury to the nearest emergency department. Here, we can elaborate on the different interventions carried out by SAMU such as administering oxygen and intravenous fluid, immobilizing the fractured limbs, and providing pain management. In combating a significant burden of emergencies, either traumatic or nontraumatic, Muhimbili National Hospital established emergency medicine as a department. ${ }^{20}$ Task shifting is a known alternative effective intervention strategy in resource-constrained settings; this was shown in a research by Chamberlain et $\mathrm{al}^{21}$ through a retrospective study done in Uganda, where nurses were trained for 2 years to become mid-level practitioners. Thus, they were providing emergency care in rural hospitals, and this reduced the mortality rate of patients consulting the emergency department for medical or traumatic conditions. ${ }^{21}$

\section{Conclusion and recommendations}

The world has seen a tremendous increase in traumatic surgical emergencies during the last decade, and this was associated with significant mortality and morbidity, although high-income countries are well prepared to face this global health concern. But in low- and middle-income countries, this burden is becoming increasingly catastrophic as RTIs are increasing at a significant rate; however, there is as yet no proportional increase in the healthcare workforce in these areas. Most emergency departments in Sub-Saharan Africa are being overcrowded, but there are some centers with limited settings with newly established local effective measures that have shown an impact in reducing traumatic emergencies-related mortality and morbidity. This review has shown that in Sub-Saharan region, in the coming years, there is a trend toward an increase in the number of RTIs, mostly in the form of musculoskeletal and traumatic brain injuries.

Hence from the study findings, in the following recommendations are provided for resource-constrained settings:

- Task shifting to shortly trained health care providers for managing emergencies in rural areas, before referral to higher level when needed.

- Establishment of a system similar to SAMU in the concerned centers. 
- Increase in the amount of research conducted on this global burden.

- Increase in the concerned workforce by training many surgeons, especially orthopedic surgeons, neurosurgeons, and general surgeons.

- Mass casualty protocol implementation and regular trainings on management of mass casualty incident.

- Zero tolerance in regard to alcohol and psychoactive substance use among drivers.

\section{Acknowledgments}

The contributions of Dr Bennie Alan, Dr Munyaneza Robert, and Dr Umugwaneza Nathalie are highly appreciated.

\section{Disclosure}

The author reports no conflicts of interest in this work.

\section{References}

1. Yamuragiye A, Ibambasi A, Mutuyimana A, Mutuyemariya O, Nsereko E. Short communication injury profile in an emergency department at a referral hospital in Kigali, Rwanda. Rwanda J Health Sci. 2013;2(1):61-62.

2. World Health Organization. Global Status Report on Road Safety 2013: Supporting a Decade of Action. Geneva: WHO; 2013.

3. World Health Organisation. Global Status Report on Road Safety 2015. Geneva: WHO; 2015.

4. Kisitu DK, Eyler LE, Kajja I, et al. The role of Ugandan District Hospital orthopedic units in the care of vulnerable road users: a cross-sectional study. Inj Epidemiol. 2016;3(1):27.

5. Afuwape OO, Ogunlade SO, Alonge T, Ayorinde OR. An audit of deaths in the emergency room in the University College Hospital Ibadan. Niger J Clin Pract. 2009;12(2):138-140.

6. Thanni LO, Kehinde OA. Trauma at a Nigerian teaching hospital: pattern and documentation of presentation. Afr Health Sci. 2006;6(2):104-107.

7. Aluisio AR, De Wult A, Louis A, Bloem C. Epidemiology of traumatic injuries in the northeast region of Haiti: a cross-sectional study. Prehosp Disaster Med. 2015;30(6):599-605.
8. Braun CT, Gnägi CR, Klukowska-Rötzler J, Ahmad SS, Ricklin ME, Exadaktylos AK. Trends and weekly cycles in a large Swiss emergency centre: a 10 year period at the University Hospital of Bern. Int J Environ Res Public Health. 2017;14(10):1239.

9. Emejulu JKC, Isiguzo CM, Agbasoga CE, Ogbuagu CN. Traumatic brain injury in the accident and emergency department of a tertiary hospital in Nigeria. East Central Afr J Surg. 2010;15(2):28-38.

10. DiMaggio C, Ayoung-Chee P, Shinseke M, et al. Traumatic injury in the United States: in-patient epidemiology 2000-2011. Injury. 2016;47(7):1393-1403.

11. Bogstrand ST, Gjerde H, Normann PT, Rossow I, Ekeberg Q. Alcohol, psychoactive substances and non-fatal road traffic accidents - a casecontrol study. BMC Public Health. 2012;12:734.

12. Aluisio AR, Umuhire OF, Mbanjumucyo G, et al. Epidemiologic characteristics of pediatric trauma patients receiving prehospital care in Kigali. Pediatr Emerg Care. Epub 2017 Feb 4.

13. Stephens T, Mezei A, O'Hara NN, et al. When surgical resources are severely constrained, who receives care? Determinants of access to orthopaedic trauma surgery in Uganda. World J Surg. 2017;41(6): $1415-1419$.

14. Elias A, Tezera C. Orthopedic and major limb trauma at the Tikur Anbessa University Hospital, Addis Ababa - Ethiopia. East Cent Afr J Surg. 2005;10(2):43-50.

15. Chekijian S, Paul M, Kohl VP, et al. The global burden of road injury: its relevance to the emergency physician. Emerg Med Int. 2014;2014: 139219.

16. Ben-Ishay O, Mitaritonno M, Catena F, Sartelli M, Ansaloni L, Kluger Y. Mass casualty incidents - time to engage. World J Emerg Surg. 2016;11:8.

17. Peden M. Global collaboration on road traffic injury prevention. Int $J$ Inj Contr Saf Promot. 2005;12(2):85-91.

18. Ng-Kamstra JS, Greenberg SLM, Abdullah F, et al. Global Surgery 2030: a roadmap for high income country actors. BMJ Glob Health. 2016;1(1):e000011.

19. Scott JW, Nyinawankusi JD, Enumah S, et al. Improving prehospital trauma care in Rwanda through continuous quality improvement: an interrupted time series analysis. Injury. 2017;48(7):1376-1381.

20. Reynolds TA, Mfinanga JA, Sawe HR, Runyon MS, Mwafongo V. Emergency care capacity in Africa: a clinical and educational initiative in Tanzania. J Public Health Policy. 2012;33(Suppl 1):S126-S137.

21. Chamberlain S, Stolz U, Dreifuss B, et al. Mortality related to acute illness and injury in rural Uganda: task shifting to improve outcomes. PLoS One. 2015;10(4):e0122559.
Open Access Emergency Medicine

\section{Publish your work in this journal}

The Open Access Emergency Medicine is an international, peerreviewed, open access journal publishing original research, reports, editorials, reviews and commentaries on all aspects of emergency medicine. The manuscript management system is completely online and includes a very quick and fair peer-review system, which is all

\section{Dovepress}

easy to use. Visit http://www.dovepress.com/testimonials.php to read real quotes from published authors. 